\title{
A note on the stationary Euler equations of hydrodynamics
}

\author{
K. Cieliebak and E. Volkov
}

\begin{abstract}
This note concerns stationary solutions of the Euler equations for an ideal fluid on a closed 3-manifold. We prove that if the velocity field of such a solution has no zeroes and real analytic Bernoulli function, then it can be rescaled to the Reeb vector field of a stable Hamiltonian structure. In particular, such a vector field has a periodic orbit unless the 3-manifold is a torus bundle over the circle. We provide a counterexample showing that the correspondence breaks down without the real analyticity hypothesis.
\end{abstract}

\section{Introduction}

The time evolution of an incompressible, inviscous ("ideal") fluid of constant density is described by the Euler equations

$$
\partial_{t} X+\nabla_{X} X=-\nabla p, \quad \operatorname{div} X=0
$$

for the velocity field $X$ of the fluid and its pressure $p$ (both time-dependent). These equations make sense on any Riemannian 3-manifold $(M, g)$ equipped with a volume form $\mu$ (not necessarily the one induced by the metric). Note that the first equation involves only the metric (via the covariant derivative and the gradient), while the second one involves only the volume form (via the divergence defined by $\left.L_{X} \mu=(\operatorname{div} X) \mu\right)$.

In this note we are interested in stationary solutions of the Euler equations, i.e., time-independent pairs $(X, p)$ satisfying the stationary Euler equations

$$
\begin{gathered}
\nabla_{X} X=-\nabla p, \\
L_{X} \mu=0 .
\end{gathered}
$$

Since by equation (11) the velocity field $X$ determines the pressure $p$ uniquely up to a constant, we will often suppress explicit mentioning of $p$ and refer to $X$ as a stationary solution of the Euler equations. Equation (11) can equivalently be written as (see e.g. 3] )

$$
\operatorname{curl} X \times X=-\nabla h
$$


where $h:=p+\frac{|X|^{2}}{2}$ is the Bernoulli function, and the curl and cross product of vector fields are defined by $d\left(i_{X} g\right)=i_{\operatorname{curl} X} \mu$ and $i_{X \times Y} g=i_{Y} i_{X} \mu$. Another equivalent way of writing equation (1), which will be particularly useful for our purposes, is in terms of the 1 -form $\lambda:=i_{X} g$ as (see e.g. [6])

$$
i_{X} d \lambda=-d h
$$

Note also that equation (2) is equivalent to closedness of the 2 -form $\omega:=i_{X} \mu$. The goal of this note is to prove the following result.

Theorem 1.1. Let $X$ be a solution of the stationary Euler equations (11), (2) with respect to a metric $g$ and volume form $\mu$ on a closed, oriented 3-manifold $M$. Suppose that $X$ has no zeroes and its Bernoulli function is real analytic (for some real analytic structure on $M$ ). Then $X$ also solves equation (1) with respect to a different metric $\widehat{g}$ such that the corresponding Bernoulli function is constant.

Let us discuss some consequences of this results.

(1) Following earlier work of Etnyre and Ghrist [7, it was observed in [6] that a vector field as in Theorem 1.1 has the following symplectic interpretation. The nowhere vanishing closed 2 -form $\omega$, the 1 -form $\lambda=i_{X} \widehat{g}$ and the rescaled vector field $\widetilde{X}=X / \widetilde{\lambda}(X)$ satisfy

$$
i_{\widetilde{X}} d \widetilde{\lambda}=i_{\widetilde{X}} \omega=0, \quad \widetilde{\lambda}(\widetilde{X})=1 .
$$

In symplectic terminology [4, this means that $(\omega, \tilde{\lambda})$ is a stable Hamiltonian structure with Reeb vector field $\widetilde{X}$. So we have shown

Corollary 1.2. A vector field $X$ as in Theorem 1.1 can be rescaled by a positive function to the Reeb vector field of some stable Hamiltonian structure.

(2) By Corollary 1.2 the 1-dimensional oriented foliation defined by $X$ has the same dynamical properties as the foliation defined by the Reeb vector field of some stable Hamiltonian structure. In particular, the proof of the Weinstein conjecture for stable Hamiltonian structures by Hutchings and Taubes 15] yields

Corollary 1.3. Let $M$ be a closed, oriented 3-manifold which is not a 2-torus bundle over the circle. Then every stationary solution of the Euler equations on $M$ with real analytic Bernoulli function possesses a zero or a periodic orbit.

This result was previously proved by Etnyre and Ghrist [7] in the case of the 3 -sphere, and combining their arguments with [15] yields a simple direct proof of Corollary 1.3. see Section 2 below.

(3) Let $\widetilde{\lambda}, \widetilde{X}$ be as in (1) and define a new metric $\widetilde{g}:=\widetilde{\lambda}(X) \widehat{g}$. Then $\widetilde{\lambda}=i_{\widetilde{X}} \widetilde{g}$, i.e., $\tilde{\lambda}$ is the dual 1-form to $\widetilde{X}$ with respect to the metric $\widetilde{g}$. Equation (5) implies that $\widetilde{X}$ satisfies equation (3) with respect to the metric $\widetilde{g}$ and constant Bernoulli 
function $\widetilde{h}$, and equation (2) with respect to the volume form $\widetilde{\mu}=\widetilde{\lambda}(X) \mu$. Moreover, the corresponding pressure $\widetilde{p}=\widetilde{h}-\widetilde{g}(\widetilde{X}, \widetilde{X}) / 2$ is constant, so we have shown

Corollary 1.4. A vector field $X$ as in Theorem 1.1] can be rescaled by a positive function to a solution of the stationary Euler equations, with respect to some new metric and volume form, such that the corresponding pressure is constant.

Geometrically, the equations $\widetilde{\nabla}_{\widetilde{X}} \widetilde{X}=0$ and $\widetilde{g}(\widetilde{X}, \widetilde{X})=1$ for the rescaled vector field $\widetilde{X}$ and the metric $\widetilde{g}$ mean that all orbits of $\widetilde{X}$ are geodesics parametrized by arclength; see [17] for further discussion of the question of geodesibility.

Corollary 1.4 somewhat resembles the description of solutions of a mechanical system with Lagrangian $L=|\dot{q}|^{2} / 2-V(q)$ and energy $E>\max V$ as geodesics of the Jacobi metric $\widetilde{g}=\sqrt{E-V(q)} g$. It would be nice to better understand its physical interpretation.

(4) Corollary 1.2 opens up the possibility of studying the dynamics of stationary solutions of the Euler equations by holomorphic curve techniques as in [13, 15], with implications for questions of hydrodynamical stability. To illustrate this line of argument, let us recall an instability criterion of Friedlander and Vishik [10]: If a stationary solution $X$ of the Euler equations possesses a hyperbolic zero or periodic orbit, then it is linearly unstable in the sense that the linearized Euler equations at $X$ have solutions whose $L^{2}$-norm grows exponentially in time. On the other hand, Hutchings and Taubes [15] proved that each Reeb vector field with nondegenerate periodic orbits on a closed 3-manifold which is not a lens space has a hyperbolc periodic orbit. Combining these two results with the arguments in [9] in the case of the 3 -torus, we obtain

Corollary 1.5. For a generic metric on a closed 3-manifold with is not a lens space, each curl eigenfield curl $X=\lambda X$ with $\lambda \neq 0$ has a hyperbolic zero or periodic orbit, and is therefore linearly unstable.

We hope that the techniques developed in this note can lead to similar instability results for more general stationary solutions of the Euler equations.

(5) Theorem 1.1 and its Corollaries 1.2 and 1.4 become false without the real analyticity hypothesis:

Proposition 1.6. There exists a smooth, nowhere vanishing vector field $X$ solving the stationary Euler equations (1), (2) on some closed, oriented 3-manifold $M$ which cannot be rescaled by a positive function to the Reeb vector field of a stable Hamiltonian structure.

The proof of Theorem 1.1 is based on the observation that equation (4) does not explicitly involve the metric. If $(X, \lambda, h)$ is a solution of this equation with $\lambda(X)>0$, then the vector field $X$ solves equation (3) with respect to any metric $g$ with $i_{X} g=\lambda$. Such a metric exists but is not unique because it can 
be defined arbitrarily on $\operatorname{ker} \lambda$. So to prove Theorem 1.1, it suffices to find a stabilizing 1-form, i.e., a 1-form $\nu$ satisfying

$$
i_{X} d \nu=0, \quad \nu(X)>0 .
$$

Using the fact that $h$ is preserved by the flow of $X$ (which an immediate consequence of equation (4)), we will construct $\nu$ in two steps. In Section 2 we construct $\nu$ near the singular level sets of $h$, and in Section 3 we extend it over the regular level sets. Proposition 1.6 will be proved in Section 4.

Acknowledgements. We thank M. Goresky for a helpful discussion on real analytic sets. Much of this research was carried out during the first author's visits to Chuo University, the Simons Center for Geometry and Physics, and the Institute for Advanced Study. He thanks them for their hospitality.

\section{Construction of $\nu$ near the singular level sets}

We begin with some properties of real analytic sets in the plane. Let $D \subset \mathbb{R}^{2}$ be the open unit disk and $h: D \rightarrow \mathbb{R}$ the restriction of a nonconstant real analytic function defined on a neighbourhood of $\bar{D}$. Let $E \subset D$ be a connected component of $h^{-1}(0)$ containing more than one point. For $x \in E$ we denote by $o(x) \geq 1$ the vanishing order of $h$ at $x$, i.e., the lowest order of a nonvanishing partial derivative of $h$ at $x$. Set $k:=\min \{o(x) \mid x \in E\}$.

Lemma 2.1. (a) $E_{1}:=\{x \in E \mid o(x)>k\}$ is a finite set and $E_{0}:=E \backslash E_{1}$ is a finite union of embedded curves.

(b) Each $x \in E_{1}$ has an open neighbourhood $U \subset D$ such that $E \cap U$ is a finite union $A_{1} \cup \cdots \cup A_{m}$ of $C^{1}$-embedded half-open intervals that meet at the $x$ and are disjoint away from $x$.

(c) Near each $x \in E_{0}$ (after possibly replacing $h$ by $-h$ if $k$ is even) the function $g=\sqrt[k]{h}$ is real analytic with $d_{x} g \neq 0$, and the level sets of $h$ form a foliation near $x$.

Proof. $E$ is a $C$-analytic set in the sense of [5]. Its $C$-rank of $E$ at $x \in E$ is defined to be the maximal number of elements in the local vanishing ideal at $x$ (consisting of the germs of holomorphic functions vanishing on $E^{*}$ ) of the minimal complex analytic set $E^{*}$ containing $E$ whose differentials at $x$ are linearly independent. By definition, the $C$-rank can only take the values $0,1,2$.

If the $C$-rank of $E$ at $x$ equals 2, then $x$ is an isolated point of $E$ by [5, Proposition 16]. Since we have assumed $E$ to be connected and to contain more than one point, this does not occur.

If the $C$-rank of $E$ equals 0 at all points in a neighbourhood of $x$, then by [5, Proposition 16] $E \subset D$ is a real analytic submanifold of dimension 2, and thus $E=D$. Since we have assumed $h$ to be nonconstant, this does not occur. 
Thus for each $x \in E$ the maximal $C$-rank of $E$ at nearby points equals 1 . Now by [5, Proposition 16] the set $E_{1}^{\prime} \subset E$ of points in $E$ of $C$-rank 0 is a proper $C$-analytic subset of $E$, hence consists of finitely many points, and the set $E_{0}^{\prime}=E \backslash E_{1}^{\prime}$ of points in $E$ of $C$-rank 1 is a 1-dimensional real analytic submanifold of $D$, hence a finite union of embedded curves. Now we can prove parts (a-c) of the lemma.

For part (a), it suffices to show $E_{0} \subset E_{0}^{\prime}$. To see this, consider $x \in E_{0}$, i.e. $o(x)=$ $k \geq 1$ as defined above. Let $\frac{\partial^{k} h}{\partial x_{1}^{i} \partial x_{2}^{j}}(x) \neq 0$ be a nonvanishing partial derivative of order $k$ at $x$. Suppose $i>0$ (the case $j>0$ is analogous). Since $k$ is the lowest order of a nonvanishing partial derivative of $h$ on $E$, the real analytic function $g:=\frac{\partial^{k-1} h}{\partial x_{1}^{i-1} \partial x_{2}^{j}}: D \rightarrow \mathbb{R}$ vanishes on $E$ and thus belongs to the vanishing ideal of $E$ at $x$. Since $\frac{\partial g}{\partial x_{1}}(x) \neq 0$, it follows that the $C$-rank of $x$ equals 1 and thus $x \in E_{0}^{\prime}$.

Next we prove (b). According to [11, Part I, Sections 1.4 and 1.7], each $x \in E_{1}$ has an open neighbourhood $U \subset D$ such that $E \cap U$ is a finite union $A_{1} \cup \cdots \cup A_{m}$ of $C^{0}$-embedded half-open intervals that meet at the $x$ and are disjoint away from $x$. That the $A_{i}$ are actually $C^{1}$-embedded is a consequence of the curve selection lemma as stated in [1, Proposition 2.2].

For part (c), consider $x \in E_{0} \subset E_{0}^{\prime}$. Since the $C$-rank of $E$ at $x$ equals 1 , the vanishing ideal of $E^{*}$ at $x$ is generated by one holomorphic function $g^{*}$ with $d_{x} g^{*} \neq 0$. (To see this, pick local holomorphic coordinates $\left(z_{1}, z_{2}\right)$ near $x$ in which $g^{*}\left(z_{1}, z_{2}\right)=z_{1}$.) The real or imaginary part of $g^{*}$ then defines a real analytic function $g: D \rightarrow \mathbb{R}$ with $d_{x} g \neq 0$ and generating the vanishing ideal of $E$ at $x$. Thus $h=g h_{1}$ for a real analytic function $h_{1}$. If $h_{1}(x)=0$, then the $C$-analytic set $h_{1}^{-1}(0)$ is contained in $E$. If $h_{1}^{-1}(0)$ agrees with $E$ near $x$ we have $h_{1}=g h_{2}$ for a real analytic function $h_{2}$. Continuing inductively, after $\ell$ steps we have $h=g^{\ell} h_{\ell}$ for a real analytic function $h_{\ell}$. Since $h$ vanishes to order $k$ at generic points of $E$, this process terminates at $h=g^{k} h_{h}$ for a real analytic function $h_{k}$ that doesn't vanish identically of $E$. Since $o(x)=k$, we have $h_{k}(x) \neq 0$. After possibly replacing $h$ by $-h$ we may assume that $h_{k}(x)>0$, and by replacing $g$ by $g \sqrt[k]{h_{k}}$ we can then achieve $h=g^{k}$. Note that if $k$ is odd, then the level sets $\{h=t\}$ near $t=0$ correspond to the level sets $\{g=\sqrt[k]{t}\}$ and thus form a foliation. If $k$ is even, then the level sets $\{h=t\}$ are empty for $t<0$ and correspond to the level sets $\{g= \pm \sqrt[k]{t}\}$ for $t \geq 0$, so again they form a foliation.

Remark 2.2. As was shown in [7] in the case of the 3-sphere, Lemma 2.1] suffices to prove Corollary 1.3. For this, consider a stationary solution $X$ of the Euler equations with real analytic Bernoulli function $h$ and without zeroes or periodic orbits. We apply Lemma 2.1 to the restriction of $h$ to a real analytic embedded disk $D \subset M$ transverse to $X$ and a connected component $E$ of $S \cap D$, where $S$ is a connected component of a level set $h^{-1}(c)$.

If $\left.h\right|_{D}$ were constant, then $h$ would be constant on $M$ by unique continuation, so $X$ would be the Reeb vector field of a stable Hamiltonian structure. Since $M$ is 
not a $T^{2}$-bundle over $S^{1}, X$ would have a periodic orbit by [15, which we have excluded by assumption. So $\left.h\right|_{D}$ is nonconstant. If a connected component $E$ consisted of a single point, the corresponding set $S$ would be a periodic orbit, so again this does not occur. Similarly, each point of the singular set $E_{1} \subset E$ would give rise to a periodic orbit (see the discussion preceding Lemma 2.4), so we must have $E_{1}=\emptyset$.

Now Lemma 2.1 (c) shows that the level sets of $h$ define a foliation of $M$ by invariant 2-tori. We claim that there exists an embedded closed curve $\gamma$ transverse to the leaves of this foliation. To see this, pick any curve $\beta: \mathbb{R} \rightarrow M$ parametrized by arclength and orthogonal to the leaves (with respect to some Riemannian metric); then $h \circ \beta: \mathbb{R} \rightarrow[\min h, \max h]$ takes some value $c$ infinitely often, and since $h^{-1}(c)$ is a union of finitely many 2 -tori, $\beta$ meets some 2-torus leaf of the foliation twice and we can close it up along this leaf to obtain $\gamma: S^{1} \rightarrow M$ transverse to the leaves. Since $M$ is connected, $\gamma$ meets every leaf and we can choose it to intersect each leaf exactly once. Assigning to $x \in M$ the value $t \in S^{1}$ for which $\gamma(t)$ lies on the leaf through $x$ defines a 2-torus bundle $T^{2} \rightarrow M \rightarrow S^{1}$, contradicting the hypothesis on $M$.

Let us now return to a solution $(X, \lambda, h)$ of the Euler equations

$$
i_{X} d \lambda=-d h, \quad L_{X} \mu=0, \quad \lambda(X)>0 .
$$

We first observe that the simplest obstruction to geodesibility in the sense of [17] vanishes:

Lemma 2.3. The foliation by flow lines of $X$ has no Reeb components.

Proof. Suppose that $Z \subset M$ is a Reeb component, i.e., an embedded cylinder invariant under the flow such that the flow direction induces the boundary orientation on both boundary circles, and all flow lines that meet the interior of $Z$ converge to the boundary in forward and backward time. Since $h$ is invariant under the flow of $X$, the last property implies that $h$ is constant on $Z$. Thus $i_{X} d \lambda=-d h$ vanishes on $Z$ and we get the contradiction

$$
0=\int_{Z} d \lambda=\int_{\partial Z} \lambda>0 .
$$

Suppose now that the Bernoulli function $h: M \rightarrow \mathbb{R}$ is real analytic. Consider a connected component $S$ of a singular level set $h^{-1}(c)$. After replacing $h$ by $h-c$ we may assume that $c=0$. For $x \in S$ we denote by $o(x) \geq 1$ the vanishing order of $h$ at $x$, i.e., the lowest order of a nonvanishing derivative of $h$ at $x$. Set $k:=\min \{o(x) \mid x \in S\}$. Since $h$ is invariant under $X$, so are the sets

$$
S_{1}:=\{x \in S \mid o(x)>k\}, \quad S_{0}:=S \backslash S_{1} .
$$


Lemma 2.4. (a) $S_{1}$ is a finite union of periodic orbits, and $S_{0}$ is a (possibly empty, possibly noncompact, possibly disconnected) embedded surface.

(b) Let $U \subset M$ be an open tubular neighbourhood of a connected component $\gamma$ of $S_{1}$, and suppose that $S_{0} \cap U \neq \emptyset$. Then $U \cap S$ is a finite union $Z_{1} \cup \cdots \cup Z_{n}$ of $C^{1}$-immersed invariant half-open cylinders whose interiors are embedded in $U \backslash \gamma$ and whose boundaries are $d$-fold coverings of $\gamma$, for some $d \in \mathbb{N}$.

Proof. We apply Lemma 2.1 to the restriction of $h$ to an embedded disk $D \subset M$ transverse to $X$ and a connected component $E$ of $S \cap D$. Then $E_{i}=S_{i} \cap D$, $i=0,1$, and part (a) follows directly from Lemma 2.1 (a).

For part (b), we choose the disk $D$ centered at $\gamma$. By Lemma 2.1 (b), $E \cap U$ is a finite union $A_{1} \cup \cdots \cup A_{m}$ of $C^{1}$-embedded arcs that meet at the origin and are disjoint outside the origin. The return map $\phi: D \rightarrow D$ of the flow of $X$ acts as a permutation on the arcs, and the orbits of this action give rise to the surfaces $Z_{1}, \ldots, Z_{n}$ (for some $n \leq m$ ). We number the $\operatorname{arcs}$ in counterclockwise order, so $\phi$ induces a permutation $\bar{\phi}$ of the set $\{1, \ldots, m\}$. Since $\phi$ is a homeomorphism, it must map adjacent arcs to adjacent arcs, so the permutation $\bar{\phi}$ satisfies $\bar{\phi}(k+1)=\bar{\phi}(k) \pm 1$ for all $k$. In other words, $\bar{\phi}$ gives an element in the orthogonal group $O(2)$ acting on the $m$-th roots of unity. Since $\phi$ preserves the area form induced by $\omega=i_{X} \mu$ on $D$, it is orientation preserving and $\bar{\phi} \in S O(2)$ is a rotation. Write $m=n d$, where $n$ is the number of orbits of $\bar{\phi}$ and $d$ the number of points in one orbit. Then each orbit corresponds to a $C^{1}$ immersed half-open cylinder whose interior is embedded and whose boundary is the $d$-fold covering of $\gamma$.

Remark 2.5. For $d=2$ in Lemma 2.4 each $Z_{i}$ is homeomorphic to an embedded open Möbius strip. This situation arises for example at negative hyperbolic orbits in Reeb flows, see e.g. [15].

According to the Arnold-Liouville theorem [2, each regular level set of $h$ is a disjoint union of finitely many embedded 2-tori on which the flow of $X$ is linear. The following proposition gives a similarly precise description of the flow on the singular level sets in the case that the Bernoulli function $h: M \rightarrow \mathbb{R}$ is real analytic.

Proposition 2.6. Let $(X, \lambda, h)$ be a solution of (6) on a closed oriented 3manifold $M$ with $h$ real analytic.

(a) The function $h: M \rightarrow \mathbb{R}$ has finitely many critical values.

(b) Each singular level set of $h$ is a finite disjoint union of embedded $X$-invariant sets that are periodic orbits, 2-tori, Klein bottles, open cylinders, or open Möbius strips. The closures of the open cylinders and Möbius strips are $C^{1}$-immersed closed cylinders and Möbius strips whose boundary components are d-fold coverings of periodic orbits, where finitely many of them can meet at the same periodic orbit.

(c) On each 2-torus as in (b) the flow is linear (rational or irrational). On each Klein bottle the flow is linear and periodic. On each Möbius strip the flow is 
periodic. On each cylinder the flow is either periodic, or all flow lines converge to the boundary in forward and backward time.

(d) On each cylinder as in (b) the flow direction induces the boundary orientation on one boundary circle, and the opposite orientation on the other one.

Proof. (a) The critical point set $C$ of $h$ is a compact analytic subset of $M$, hence its image $h(C)$ is a compact semi-analytic subset of $\mathbb{R}$. Since every compact semi-analytic subset of $\mathbb{R}$ is a finite union of points and closed intervals, and $h(C)$ contains no intervals by Sard's theorem, the set $h(C)$ is finite.

(b) Consider a connected component $S$ of a singular level set $h^{-1}(c)$ and define $S_{0}, S_{1}$ as above. By Lemma 2.4 (a), $S_{1}$ is a finite union of periodic orbits and each connected component $\stackrel{\circ}{Z}$ of $S_{0}$ is an embedded surface. Since $X$ is tangent to $\stackrel{\circ}{Z}$, the Euler characteristic of $\dot{Z}$ vanishes, so $Z^{\circ}$ can only be a torus, Klein bottle, open cylinder, or open Möbius strip. By Lemma 2.4 (b), the closure $Z$ of $Z$ is a $C^{1}$-immersed closed cylinder resp. Möbius strip whose boundary components belong to are $d$-fold coverings of periodic orbits in $S_{1}$. Since only finitely many cylinders and Möbius strips meet at each orbit in $S_{1}$, the number of components of $S_{0}$ is finite.

(c) We continue in the notation from (b). Lemma 2.1 (c) shows that near each $x \in S_{0}$ (after possibly replacing $h$ by $-h$ if $k$ is even) we can write $h=g^{k}$ for a real analytic function $g$ near $x$. Now we distinguish 2 cases.

Case 1: $k$ is odd.

Then $S_{0}$ is a regular level set of the real analytic $X$-invariant function $g=\sqrt[k]{h}$ defined on a neighbourhood of $S_{0}$. In particular, $S_{0}$ is orientable, so the closure $Z$ of each connected component is either a torus or a cylinder. By the ArnoldLiouville theorem 2, on each torus the flow is linear. For a cylinder, the flow preserves the smooth area form $\mu / d g$ on $Z^{\circ}$ (where $\mu$ is the invariant volume form on $M$, and the total area may be infinite). Thus the return map on each local transverse slice preserves a smooth measure. This implies that the flow is either periodic, or all flow lines on $Z^{\circ}$ converge to $\partial Z$ in forward and backward time.

Case 2: $k$ is even.

Consider again the closure $Z$ of a connected component of $S_{0}$. If $Z$ is orientable, then so is its normal bundle in $M$, hence we can choose a $k$-th root $g=\sqrt[k]{h}$ near $\dot{Z}$ (requiring it to be positive on one side of $Z$ ) and proceed as in Case 1. If $Z$ is non-orientable, consider its orientable $2-1$ covering $\widetilde{Z} \rightarrow Z$. Pull back the normal bundle to $Z$ to obtain an orientable 2-1 covering $\widetilde{U} \rightarrow U$ of a neighbourhood $U$ of $Z$ in $M$. In particular, the normal bundle of $\widetilde{Z}$ in $\widetilde{U}$ is trivial, so we can choose a $k$-th root $\widetilde{g}=\sqrt[k]{\widetilde{h}}$ of the pullback $\widetilde{h}$ of $h$ near the interior of $\widetilde{Z}$ and proceed as in Case 1 on the covering. If $Z$ is a Klein bottle, then $\widetilde{Z}$ is a torus on which the flow is linear. Since the data on $\widetilde{Z}$ were invariant under the covering involution, the linearizing coordinates on $\widetilde{Z}$ can be chosen to descend to coordinates on $Z$ in which the flow is linear. It is easy to see that 
every linear foliation of the Klein bottle is periodic. If $Z$ is a Möbius strip, then $\widetilde{Z}$ is a cylinder on which the flow is either periodic, or all flow lines converge to $\partial \widetilde{Z}$ in forward and backward time. Now the foliation on $\widetilde{Z}$ defined by the flow is invariant under the covering involution, which in suitable coordinates on $\widetilde{Z} \cong \mathbb{R} / \mathbb{Z} \times[-1,1]$ is given by $(x, y) \mapsto(x+1 / 2,-y)$. This excludes convergence of flow lines to $\partial \widetilde{Z}$ in forward and backward time, so the flow on $\widetilde{Z}$ is periodic and descends to a periodic flow on $Z$.

(d) follows directly from Lemma 2.3 .

Example 2.7. On $\mathbb{R}^{3}$ with coordinates $(x, y, z)$ consider a solution of (6) given by

$$
X=\partial_{x}, \quad \lambda=h(z) d z, \quad \omega=d x \wedge d y, \quad \mu=d x \wedge d y \wedge d z
$$

with a positive Bernoulli function $h(z)$. As noted in the Introduction, this yields a solution of $\nabla_{X} X=-\nabla p$ with $p(z)=h^{\prime}(z) / 2$ for any metric satisfying $i_{X} g=\lambda$, i.e.,

$$
\left|\partial_{x}\right|^{2}=h(z), \quad\left\langle\partial_{x}, \partial_{y}\right\rangle=\left\langle\partial_{x}, \partial_{z}\right\rangle=0 .
$$

Note that $\operatorname{curl} X=h^{\prime}(z) \partial_{y}$. An example of such a metric with $\operatorname{vol}_{g}=d x \wedge d y \wedge d z$ is

$$
g=h(z) d x^{2}+d y^{2}+h(z)^{-1} d z^{2} .
$$

Consider now the closed oriented 3-manifold $M:=\mathbb{R}^{3} / \sim$ obtained by dividing out the equivalence relation generated by

$$
(x, y, z) \sim(x, y+1, z) \sim(x, y, z+1) \sim(x+1,-y,-z) .
$$

It is doubly covered by the 3 -torus via the map

$$
T^{3}=\mathbb{R}^{3} / \mathbb{Z}^{3} \rightarrow M, \quad[x, y, z] \mapsto[2 x, y, z] .
$$

One can also view $M$ as the mapping torus of the map

$$
T^{2} \rightarrow T^{2}, \quad(y, z) \mapsto(-y,-z) .
$$

The data $X, \lambda, \omega, \mu, g$ above descend to $M$ provided the function $h$ satisfies

$$
h(z)=h(-z)=h(z+1) .
$$

An example of such a function is $h(z)=2-\cos (2 \pi z)$. The level sets $\{z \in \mathbb{Z}\}$ and $\left\{z \in \frac{1}{2}+\mathbb{Z}\right\}$ are Klein bottles, and all other level sets are 2-tori on which the flow of $X$ is linear and periodic. This can be seen from the map

$$
T^{2} \rightarrow\{z \in \pm c+\mathbb{Z}\} \subset M, \quad[x, y] \mapsto[2 x, y, c]
$$

which is a 2-1 covering if $c \in \mathbb{Z}$ or $z \in \frac{1}{2}+\mathbb{Z}$, and a diffeomorphism otherwise. One can generalize this example to mapping tori of the shear maps

$$
(y, z) \mapsto(y+\ell z, z) \text { or }(y, z) \mapsto(-y+\ell z,-z), \quad \ell \in \mathbb{Z} .
$$

This example shows that Klein bottles (and thus also Möbius strips) can actually occur in singular level sets. 
Now we construct a stabilizing 1-form $\nu$ near the singular level sets. We assign a covering number $d_{\gamma}$ to each simple periodic orbit $\gamma$ on a singular level set as follows. If $\gamma \subset S_{0}$ has nontrivial normal bundle in $S_{0}$ we let $d_{\gamma}:=2$ (this occurs for the central orbit on a Möbius strip and the two special orbits on a Klein bottle). If $\gamma \subset S_{1}$ is $d$-fold covered by the boundary of a component in $S_{0}$ (as in Lemma 2.4) we set $d_{\gamma}:=d$. In all other cases we set $d_{\gamma}:=1$.

Proposition 2.8. There exist a closed 1-form $\nu$ on a neighbourhood of the union of the singular level sets of $h$ satisfying $\nu(X)>0$, and normalized such that $\int_{\gamma} \nu=1 / d_{\gamma}$ for each simple periodic orbit $\gamma$ on a singular level set.

Remark 2.9. We still have the freedom to multiply $\nu$ by a positive constant on each component of a singular level set that is either an isolated periodic orbit or a torus with irrational flow.

Proof. In view of Proposition 2.6 (a), it suffices to consider one connected component $S$ of a singular level set $h^{-1}(c)$ at a time. If $S$ consists only of an isolated periodic orbit $\gamma$ we pick a closed 1-form $\nu$ near $\gamma$ with $\nu(X)>0$ and $\int_{\gamma} \nu=1$.

If $S$ is a torus or a Klein bottle, then the flow defines a linear foliation on $S$. Pick a transverse linear foliation $\mathcal{F}$ and define a closed 1-form $\nu$ on $S$ by $\nu(X):=a$ and $\left.\nu\right|_{T \mathcal{F}}=0$, for some constant $a>0$. Then extend $\nu$ to a closed 1-form on a neighbourhood of $S$ in $M$. If the flow is periodic on $S$ with minimal period $T$ we choose $a=1 / T$ to satisfy the normalization condition $\int_{\gamma} \nu=1 / d_{\gamma}$. On a cylinder with irrational flow we can choose $a$ arbitrarily (this freedom will still be needed later).

It remains to consider the case that $S$ is a union of cylinders and Möbius strips whose boundaries meet in a collection $S_{1}$ of periodic orbits. We first pick a closed 1-form $\nu_{1}$ on a neighbourhood of $S_{1}$ in $M$ with $\nu_{1}(X)>0$. Using Lemma 2.4. we can normalize $\nu_{1}$ on each periodic orbit $\gamma$ in $S_{1}$ by $\int_{\gamma} \nu_{1}=1 / d_{\gamma}$. Next we consider a cylinder or Möbius strip $Z$ in $S$. The chosen form $\nu_{1}$ provides a closed 1-form near $\partial Z$ with $\nu_{1}(X)>0$. Our normalization implies that the integral of $\nu_{1}$ over each boundary component of $Z$ (oriented by $X$ ) equals 1 .

Claim: There exists an embedded $\operatorname{arc} \delta$ in $Z$ which starts and ends on $\partial Z$ and is transverse to the foliation by $X$-orbits.

To see this, consider first a cylinder $Z$ on which the flow is periodic. Then we find a submersion $\tau: Z \rightarrow[-1,1]$ (the projection onto the space of leaves) having the $X$-orbits as regular level sets, and any curve $\delta:[-1,1] \rightarrow Z$ with $\tau \circ \delta(t)=t$ has the desired properties.

Next consider a Möbius strip $Z$. By Proposition 2.6(b), the flow on $Z$ is periodic and thus defines a foliation with closed leaves. Leaves of this foliation can only represent the homology classes $c$ or $2 c$, where $c$ is the generator of $H_{1}(Z ; \mathbb{Z})$ such that $\partial Z$ oriented by $X$ represents the class $2 c$. Now embedded curves in class $c$ have nontrivial normal bundle, while embedded curves in class $2 c$ have trivial normal bundle. It follows that not all leaves can lie in class $2 c$, since otherwise the foliation would be cooriented and thus foliate a cylinder rather 
than a Möbius strip. On the other hand, since any two closed curves in class $c$ intersect, there can be at most one leaf in class $c$. Hence there is exactly one leaf $\gamma$ in class $c$. Pick an arc $\delta_{0}$ intersecting transversely a closed invariant neighbourhood $A$ of $\gamma$. Since $Z \backslash \AA$ is a cylinder foliated by closed leaves, we can connect the endpoints of $\delta_{0}$ to $\partial Z$ by two disjoint arcs transverse to the foliation to obtain the desired $\operatorname{arc} \delta$.

Finally, consider a cylinder $Z$ on which each orbit converges to $\partial Z$ in forward and backward time. Denote by $\partial_{+} Z$ (resp. $\partial_{-} Z$ ) the boundary component whose boundary orientation is induced by $X$ (resp. $-X$ ). This is possible in view of Proposition 2.6 (d). Pick a short $\operatorname{arc} \delta_{1}$ starting on $\partial_{-} Z$ and transverse to the foliation. Recall that $-X$ defines the boundary orientation on $\partial_{-} Z$, which means that $(\eta,-X)$ is a positive basis for an outward pointing vector $\eta$ at $\partial_{-} Z$. Since $\dot{\delta}_{1}$ is inward pointing, $\left(\dot{\delta}_{1}, X\right)$ is a positive basis along $\delta_{1}$. We extend $\delta_{1}$ to an arc $\delta_{2}$ ending close to $\partial_{+} Z$ by moving almost parallel to the backward $X$-orbit starting from the endpoint of $\delta_{1}$, at a slight angle to make $\delta_{2}$ transverse to the foliation. It follows that $\left(\dot{\delta}_{2}, X\right)$ is a positive basis at the endpoint of $\delta_{2}$. Since $(\eta, X)$ is also a positive basis for an outward pointing vector $\eta$ at $\partial_{+} Z$, we can connect the endpoint of $\delta_{2}$ to $\partial_{+} Z$ to obtain the desired $\operatorname{arc} \delta$. This proves the claim.

We can choose the $\operatorname{arc} \delta$ in the claim such that $\nu_{1}(\dot{\delta})=0$ near $\partial Z$. By abuse of notation, we will denote the image of $\delta$ in $Z$ again by the letter $\delta$. For $x \in Z \backslash \delta$, we denote by $\gamma_{x}$ the $X$-orbit through $x$ starting and ending on $\delta$ (oriented by $X)$. Since $\nu_{1}$ is closed, normalized by $\int_{\gamma} \nu_{1}=1$ for each boundary orbit $\gamma$ (oriented by $X$ ), and $\nu_{1}(\dot{\delta})=0$, it follows from Stokes' theorem that $\int_{\gamma_{x}} \nu_{1}=1$ for all $x$ sufficiently close to $\partial Z$.

We extend $\nu_{1}$ to a (not necessarily closed) 1 -form $\nu_{2}$ on $Z$ satisfying

(i) $\nu_{2}(X)>0$,

(ii) $\nu_{2}=\nu_{1}$ near $\partial Z$,

(iii) $\int_{\gamma_{x}} \nu_{2}=1$ for all $x \in Z \backslash \delta$.

We define a function $\phi: Z \rightarrow \mathbb{R} / \mathbb{Z}$ by $\left.\phi\right|_{\delta}:=0$ and $\phi(x):=\int_{\gamma_{x}^{-}} \nu_{2}$ for $x \notin \delta$, where $\gamma_{x}^{-}$is the part of $\gamma_{x}$ starting on $\delta$ and ending at $x$. Property (iii) shows that $\phi$ is well-defined and smooth as a map to $\mathbb{R} / \mathbb{Z}$. We claim that the closed 1-form $\nu:=d \phi$ on $Z$ agrees with $\nu_{1}$ near $\partial Z$ and satisfies $\nu(X)>0$.

For the first statement, recall that $\nu_{1}$ is closed on a tubular neighbourhood $U$ of $\partial Z$. In view of (ii), this implies that $\phi(x)=\int_{\gamma} \nu_{1} \in \mathbb{R} / \mathbb{Z}$ for $x \in U$ and $\gamma$ any path in $U$ from $\delta$ to $x$. A standard calculation shows that $\nu=d \phi=\nu_{1}$ on $U$. The second statement follows directly from (i) and the definition of $\phi$, which implies that $\nu(X)=d \phi(X)=\nu_{2}(X)>0$.

To conclude the proof of Proposition 2.8, we extend $\nu$ to a closed form on a neighbourhood of $Z$ in $M$ which agrees with $\nu_{1}$ near $\partial Z$. Performing this for 
all cylinders and Möbius strips in $S$, we obtain an extension of $\nu_{1}$ to a closed 1 -form $\nu$ on a neighbourhood of $S$ with $\nu(X)>0$. By construction $\nu$ satisfies the normalization condition $\int_{\gamma} \nu=1 / d_{\gamma}$.

The proof of Proposition 2.8 yields

Corollary 2.10. If in Theorem 1.1 the Bernoulli function is nonconstant, then $M$ is the union of finitely many connected Seifert manifolds glued along their torus boundary components.

Proof. The proof of Lemma 2.4 shows that each component $\gamma$ of $S_{1}$ in a component $S=S_{0} \cup S_{1}$ of a singular level set of $h$ has a tubular neighbourhood $U_{\gamma}$ such that $S \cap U_{\gamma}$ consists of $n C^{1}$-immersed half-open cylinders whose boundaries are $d_{\gamma}$-fold coverings of $\gamma$. Moreover, the return map on a disk $D$ transverse to $\gamma$ acts as a rotation $i \mapsto i+p$ on the set of $m=n d_{\gamma}$ arcs formed by $D \cap S$, where $\operatorname{gcd}(p, m)=n$. It follows that $U_{\gamma}$ is the mapping torus of a rotation of $D$ by an angle $2 \pi p / m$, thus $U_{\gamma}$ is Seifert fibered with a circle action going $d_{\gamma}$ times along the mapping torus and with exceptional orbit $\gamma$.

If $Z$ is the closure of a cylinder or Möbius strip in $S_{0}$, then the circle action near its boundary can be extended to a circle action on a neighbourhood of $Z$ in $M$ preserving $Z$. Tori or Klein bottles in $S_{0}$ also have neighbourhoods with circle actions preserving them. Altogether, we find a circle action on a closed neighbourhood $U$ of the union $N$ of the singular level sets, which is free except for the orbits with covering number $d_{\gamma}>1$ arising in $S_{1}$ or as exceptional orbits on Möbius strips and Klein bottles. Thus each component of $U$ is a Seifert manifold whose boundary is a union of 2-tori. Since all these 2-tori are pairwise connected by integrable regions $(a, d) \times T^{2}$ (see Section 3), we obtain the manifold $M$ by gluing together these pairs of 2 -tori.

Remark 2.11. (i) The gluing of the boundary 2-tori need not match the directions of the circle actions, so the manifold $M$ itself need not be Seifert fibered. In 3-manifold terminology, if $M$ is irreducible, then its JSJ decomposition contains no atoroidal pieces, and its decomposition according to the geometrization conjecture contains only pieces with spherical geometry.

(ii) If the singular level sets of $h$ consist only of 2-tori, then $M$ is the mapping torus of a shear map $(y, z) \mapsto(y+\ell z)$ on $T^{2}$ for some $\ell \in \mathbb{Z}$.

(iii) If in Theorem 1.1 the Bernoulli function is constant, then $d \lambda=f \omega$ for a function $f: M \rightarrow \mathbb{R}$ which is again invariant under the flow of $X$. If $f$ is real analytic and nonconstant, then again the conclusions of Corollary 2.10 hold. If $f$ equals a constant $c$, then $X$ is either the Reeb vector field of a (positive or negative) contact structure (if $c \neq 0$ ), or the horizontal vector field of a mapping torus (if $c=0$ ). See [6] for further discussion. 


\section{$3 \quad$ Extension of $\nu$ over the regular level sets}

According to Proposition 2.8, there exist a closed 1-form $\nu$ on an open neighbourhood $U$ of the union $N$ of the singular level sets of $h$ satisfying $\nu(X)>0$, and normalized such that $\int_{\gamma} \nu=1 / d_{\gamma}$ for each simple periodic orbit $\gamma$ on a singular level set. We fix such $\nu$ for the remainder of this section. Consider a connected component $V$ of $M \backslash N$. By the Arnold-Liouville theorem (2, see also [3] $), V$ is diffeomorphic to $(a, d) \times T^{2}$ such that $h(r, x)=r$ and the vector field $X$ is constant on each torus $\{r\} \times T^{2}$. The following lemma allows us to interpolate between two stabilizing closed 1-forms on such an integrable region $V$.

Lemma 3.1. Let $\omega$ be a nowhere vanishing closed 2 -form on $[a, b] \times T^{2}$ with vector field $X$ generating $\operatorname{ker} \omega$ and tangent to the tori $\{r\} \times T^{2}$. Let $\nu_{0}, \nu_{1}$ be two closed 1 -forms with $\nu_{i}(X)>0$ and $\left[\nu_{0}\right]=\left[\nu_{1}\right] \in H^{1}\left([a, b] \times T^{2} ; \mathbb{R}\right)$. Then there exists a closed 1 -form $\widetilde{\nu}$ with $\widetilde{\nu}(X)>0$ which agrees with $\nu_{0}$ near $\{a\} \times T^{2}$ and with $\nu_{1}$ near $\{b\} \times T^{2}$.

Proof. Since $\left[\nu_{0}\right]=\left[\nu_{1}\right]$, we can write $\nu_{1}=\nu_{0}+d g$ for some function $g$. Pick a cutoff function $\phi:[a, b] \rightarrow[0,1]$ which equals 0 near $a$ and 1 near $b$. We denote by $r$ the coordinate on $[a, b]$, viewed as a function on $[a, b] \times T^{2}$. Then the 1 -form

$$
\widetilde{\nu}:=\nu_{0}+d(\phi(r) g)
$$

is closed and agrees with $\nu_{0}$ near $\{a\} \times T^{2}$, and with $\nu_{1}$ near $\{b\} \times T^{2}$. Using $d g=\nu_{1}-\nu_{0}$, we can rewrite $\widetilde{\nu}$ as

$$
\widetilde{\nu}=\nu_{0}+\phi(r) d g+g \phi^{\prime}(r) d r=(1-\phi(r)) \nu_{0}+\phi(r) \nu_{1}+g \phi^{\prime}(r) d r .
$$

Since $\nu_{i}(X)>0$ and $d r(X)=0$, we see that

$$
\widetilde{\nu}(X)=(1-\phi(r)) \nu_{0}(X)+\phi(r) \nu_{1}(X)>0,
$$

so $\widetilde{\nu}$ has the desired properties.

Pick values $a<b<c<d$ such that the form $\nu$ is defined near

$$
V_{0}=((a, b] \cup[c, d)) \times T^{2} .
$$

Let $\bar{\nu}$ be the $T^{2}$-invariant 1-form on $V_{0}$ obtained by averaging $\nu$ over the tori $\{r\} \times T^{2}$. It is closed, represents the same cohomology class as $\nu$, and still satisfies $\bar{\nu}(X)>0$ (due to $T^{2}$-invariance of $X$ ). By Lemma 3.1, we find a closed 1-form $\widetilde{\nu}$ on $V_{0}$ with $\widetilde{\nu}(X)>0$ which agrees with $\nu$ near $\{a, d\} \times T^{2}$ and with $\bar{\nu}$ near $\{b, c\} \times T^{2}$.

Suppose first that the direction of the vector field $X$ is not constant in $r \in(a, d)$. By choosing $b, c$ sufficiently close to $a, d$ we can arrange that the direction of $X$ is not constant in $r \in(b, c)$. Then Proposition 3.14 in [6] provides an extension of $\widetilde{\nu}$ to a stabilizing 1-form over $V$. 
Next suppose that the direction of the vector field $X$ is constant in $r \in(a, d)$. In this case, there exists an obstruction to extending $\widetilde{\nu}$ to a stabilizing 1 -form over $V$. To describe it, we write

$$
X=\rho(r) \bar{X}
$$

for a constant vector field $\bar{X}$ on $T^{2}=\mathbb{R}^{2} / \mathbb{Z}^{2}$. For each $r \in(a, d)$ we define a 1-current on $V$ by

$$
c_{r}(\alpha):=\int_{\{r\} \times T^{2}} \alpha(\bar{X}) d \theta d \phi
$$

for a 1 -form $\alpha$. Thus $c_{r}(\alpha)$ is the pairing of the average of $\alpha$ over $\{r\} \times T^{2}$ with the vector $\bar{X}$. It is shown in Lemma 3.3 below that $c_{r}(\nu)$ equals a constant $c_{-}(\nu)$ for $r \in(a, b)$ and a constant $c_{+}(\nu)$ for $r \in(c, d)$, and $c_{-}(\nu)=c_{+}(\nu)$ is a necessary condition for extending $\widetilde{\nu}$ to a stabilizing 1-form over $V$. On the other hand, this condition is also sufficient according to Lemma 3.10 in [6]. Performing this construction for all components $V$, Theorem 1.1 follows once we can show that this condition can always be satisfied, which is the content of

Proposition 3.2. We can choose the 1-form $\nu$ in Proposition 2.8 such that $c_{-}(\nu)=c_{+}(\nu)$ for all integrable regions $V \subset M \backslash N$ on which the direction of $X$ is constant.

The proof of this proposition is based on an analysis of the currents $c_{r}$. Throughout the following discussion, we always need to distinguish the following two cases.

Case 1: The direction of $\bar{X}$ is rational.

In this case, we normalize $\bar{X}$ and choose coordinates $(\theta, \phi)$ on $T^{2}=\mathbb{R}^{2} / \mathbb{Z}^{2}$ such that $\bar{X}=\partial_{\phi}$, so the periodic orbits of $\bar{X}$ are the circles $\{(r, \theta)\} \times S^{1}$ and have period 1. It follows that

$$
c_{r}(\alpha)=\int_{0}^{1}\left(\int_{\{(r, \theta)\} \times S^{1}} \alpha\right) d \theta .
$$

Case 2: The direction of $\bar{X}$ is irrational.

In this case, since an irrational linear flow on the torus is ergodic, as a direct consequence of Birkhoff's ergodic theorem we have

$$
c_{r}(\alpha)=\lim _{T \rightarrow \infty} \frac{1}{T} \int_{0}^{T} \alpha(\dot{\gamma}(t)) d t
$$

for each orbit $\gamma$ of $\bar{X}$ on $\{r\} \times T^{2}$.

Lemma 3.3. In both cases, the currents $c_{r}$ have the following properties.

(a) $c_{r}$ is closed and invariant under $\bar{X}$.

(b) The homology class $\left[c_{r}\right] \in H_{1}\left(T^{2} ; \mathbb{R}\right)$ is independent of $r$. 
(c) $c_{r}(\widetilde{\nu})$ is independent of $r$ for each 1-form $\widetilde{\nu}$ satisfying $i_{X} d \widetilde{\nu}=0$.

(d) For all $r_{1}, r_{2}$ and $\lambda$ satisfying $i_{X} d \lambda=-d h(=-d r)$,

$$
c_{r_{2}}(\lambda)-c_{r_{1}}(\lambda)=\int_{r_{1}}^{r_{2}} \frac{1}{\rho(r)} d r
$$

Proof. We prove everything in Case 2; the proof in Case 1 is similar but easier, using formula (8) instead of (9).

(a) For closedness, note that for each function $f: V \rightarrow \mathbb{R}$ we have

$\partial c_{r}(f)=c_{r}(d f)=\lim _{T \rightarrow \infty} \frac{1}{T} \int_{0}^{T} d f(\dot{\gamma}(t)) d t=\lim _{T \rightarrow \infty} \frac{1}{T}(f(\gamma(T))-f(\gamma(0))) d t=0$

because the term $f(\gamma(t))-f(\gamma(0))$ is bounded uniformly in $T$. For invariance, let $\phi_{\tau}$ be the flow of $\bar{X}$. Then we get

$$
c_{r}\left(\phi_{\tau}^{*} \alpha\right)=\lim _{T \rightarrow \infty} \frac{1}{T} \int_{\gamma([0, T])} \phi_{\tau}^{*} \alpha=\lim _{T \rightarrow \infty} \frac{1}{T} \int_{\gamma([\tau, T+\tau])} \alpha=c_{r}(\alpha) .
$$

(b) For $r_{1}<r_{2}$ we define the 2-current

$$
C(\beta):=\lim _{T \rightarrow \infty} \frac{1}{T} \int_{r_{1}}^{r_{2}} \int_{0}^{T} \beta\left(\partial_{r}, \dot{\gamma}(t)\right) d r d t=\int_{r_{1}}^{r_{2}} c_{r}\left(i_{\partial_{r}} \beta\right) d r .
$$

Then we get

$$
\begin{aligned}
\partial C(\alpha) & =\lim _{T \rightarrow \infty} \frac{1}{T} \int_{\left[r_{1}, r_{2}\right] \times \gamma([0, T])} d \alpha \\
& =\lim _{T \rightarrow \infty} \frac{1}{T}\left\{\int_{\left[r_{1}, r_{2}\right] \times \gamma(0)} \alpha+\int_{\left\{r_{2}\right\} \times \gamma([0, T])} \alpha-\int_{\left[r_{1}, r_{2}\right] \times \gamma(T)} \alpha-\int_{\left\{r_{1}\right\} \times \gamma([0, T])} \alpha\right\} \\
& =c_{r_{2}}(\alpha)-c_{r_{1}}(\alpha)
\end{aligned}
$$

because the first and third term in the big bracket are bounded uniformly in $T$.

(c) From the proof of (b) and $i_{X} d \widetilde{\nu}=0$ we get

$$
c_{r_{2}}(\widetilde{\nu})-c_{r_{1}}(\widetilde{\nu})=\lim _{T \rightarrow \infty} \frac{1}{T} \int_{r_{1}}^{r_{2}} \int_{0}^{T} d \widetilde{\nu}\left(\partial_{r}, \dot{\gamma}(t)\right) d r d t=0
$$

because the integrand vanishes identically.

(d) From $i_{X} d \lambda=-d r$ and $\dot{\gamma}=\bar{X}=\frac{1}{\rho} X$ we get

$$
d \lambda\left(\partial_{r}, \dot{\gamma}(t)\right)=-\frac{1}{\rho(r)}\left(i_{X} d \lambda\right)\left(\partial_{r}\right)=\frac{1}{\rho(r)} d r\left(\partial_{r}\right)=\frac{1}{\rho(r)},
$$

and thus from the proof of (b),

$$
c_{r_{2}}(\lambda)-c_{r_{1}}(\lambda)=\lim _{T \rightarrow \infty} \frac{1}{T} \int_{r_{1}}^{r_{2}} \int_{0}^{T} d \lambda\left(\partial_{r}, \dot{\gamma}(t)\right) d r d t=\int_{r_{1}}^{r_{2}} \frac{1}{\rho(r)} d r .
$$


Property (a) means that $c_{r}$ it is a foliation cycle in the sense of [16] supported on $\{r\} \times T^{2}$. For a smooth function $f: V \rightarrow \mathbb{R}$ we denote its average over $\{r\} \times T^{2}$ by

$$
\bar{f}(r):=\int_{\{r\} \times T^{2}} f d \theta d \phi .
$$

Let $\nu$ be the closed 1-form on $V_{0} \subset V$ with $\nu(X)>0$ from above and define $c_{-}=c_{-}(\nu):=c_{r}(\nu)$ for $r \in(a, b)$. Then for $r \in(a, b)$,

$$
0<c_{-} \equiv c_{r}(\nu)=\overline{\nu(\bar{X})}(r)=\frac{1}{\rho(r)} \overline{\nu(X)}(r) .
$$

Since $\nu(X)$ is bounded away from 0 and $\infty$, so is $\overline{\nu(X)}(r)$, and hence by the preceding line also $\rho(r)$. Since

$$
c_{r}(\lambda)=\overline{\lambda(\bar{X})}(r)=\frac{1}{\rho(r)} \overline{\lambda(X)}(r)
$$

and $\lambda(X)$ is bounded away from 0 and $\infty$, so are $\overline{\lambda(X)}(r)$ and $c_{r}(\lambda)$.

Lemma 3.4. Let $V \subset M \backslash N$ be an integrable region on which the direction of $\bar{X}$ is constant and let $S=\bar{V} \cap h^{-1}(a)$ or $S=\bar{V} \cap h^{-1}(d)$.

(a) If the direction of $\bar{X}$ is rational, then $S$ is one of the following:

(i) an isolated periodic orbit,

(ii) a rational torus or a Klein bottle,

(iii) a union of periodic cylinders and Möbius strips connected at their (possibly multiply covered) boundaries.

(b) If the direction of $\bar{X}$ is irrational, then $S$ is one of the following:

(i) an isolated periodic orbit,

(ii) an irrational torus.

Proof. We consider the case $S=\bar{V} \cap h^{-1}(a)$, the other one being analogous.

In case (a), the flow of $X$ is periodic on each $\{r\} \times T^{2}$ for $r>a$, with uniformly bounded period. Thus for each $p \in S$ we find a sequence of periodic orbits $\gamma_{n}:\left[0, T_{n}\right] \rightarrow\left\{r_{n}\right\} \times T^{2}$ with $\gamma_{n}(0) \rightarrow p$ as $n \rightarrow \infty$ and uniformly bounded periods $T_{n}$. By the Arzela-Ascoli theorem, a subsequence of these periodic orbits converges to a periodic orbit through $p$. This shows that the flow is also periodic on $S$, so $S$ must be of one of the types (i)-(iii) described in (a) of the lemma.

Consider now case (b) in which the direction of $\bar{X}$ is irrational. It follows from $X=\rho \bar{X}$ and (7) that for $r>a$ the currents $c_{r}$ are given by

$$
c_{r}(\alpha)=\int_{M} \alpha(X) d \mathfrak{m}_{r}
$$


for Borel measures $\mathfrak{m}_{r}$ supported on $\{r\} \times T^{2}$ and invariant under the flow of $X$. Moreover, the total mass of these measures is uniformly bounded away from 0 and $\infty$. By the Banach-Alaoglu theorem (viewing the space of Borel measures as dual to the space of continuous functions with the weak* topology), there exists a sequence $r_{n} \searrow a$ such that the $\mathfrak{m}_{r_{n}}$ converge in the weak ${ }^{*}$ topology to a nontrivial invariant Borel measure $\mathfrak{m}$ supported on $S$. Recall that weak ${ }^{*}$ convergence means that for each continuous 1-form $\alpha$ defined on a neighbourhood of $S$ in $V \cup S$ (and arbitrarily extended to $M$ ),

$$
\lim _{n \rightarrow \infty} c_{r_{n}}(\alpha)=\int_{M} \alpha(X) d \mathfrak{m}
$$

Using this, we will now finish the proof in two steps.

Step 1. We will first show that $S_{0}$ cannot contain any periodic orbit. Arguing by contradiction, suppose that $S_{0}$ carries a periodic orbit $\delta_{a}$. Then by Proposition 2.6 a tubular neighbourhood $(-\varepsilon, \varepsilon) \times S^{1}$ of $\delta_{a}=\{0\} \times S^{1}$ in $S_{0}$ is foliated by periodic orbits $\{x\} \times S^{1}$. Pick a nondecreasing function $f:(-\varepsilon, \varepsilon) \rightarrow[-1,1]$ which equals \pm 1 near $\pm \varepsilon$ and has the regular level set $\{0\}=f^{-1}(0)$. We extend $f$ by projection onto the first factor to a function on $(-\varepsilon, \varepsilon) \times S^{1}$, and from there by another projection to a function on a neighbourhood $[a, b) \times(-\varepsilon, \varepsilon) \times S^{1}$ in $V \cup S$ on which $h(r, x, y)=h(r)$ and $f(r, x, y)=f(x, y)$. (Here we use Lemma 2.1(c), which asserts that the level sets of $h$ form a foliation near $\delta_{a}$.) The exact 1-form $d f$ on this set vanishes near $[a, b) \times\{ \pm \varepsilon\} \times S^{1}$, so it can be extended by zero to a closed 1-form $\alpha$ on $h^{-1}([a, b))$. By construction we have $\alpha(X) \equiv 0$ on $S$ and hence $\int_{M} \alpha(X) d \mathfrak{m}=0$. On the other hand, the direction of $\bar{X}$ is irrational for $r>a$, so we can arrange that the trajectories of $\bar{X}$ on $\{r\} \times T^{2}$ are transverse to the closed curve $\delta_{r}:=f^{-1}(0) \cap\{r\} \times T^{2}$ for all $r>a$. Since $\left.\alpha\right|_{\delta_{r}}=0$, it follows that $\frac{1}{T} \int_{0}^{T} \alpha(\dot{\gamma}) d t=\frac{1}{T} \int_{0}^{T} d f(\dot{\gamma}) d t= \pm \frac{2}{T}$, where $T$ is the time in which a trajectory $\gamma$ of $\bar{X}$ starting from $\delta_{r}$ hits it again for the first time. By averaging over the trajectories we obtain $c_{r}(\alpha)=c \neq 0$ for all $r>a$, where the constant $c$ does not depend on $r$ by closedness of $\alpha$ and Lemma 3.3.(c). But this contradicts the convergence in (10).

Step 2. The preceding step excludes that $S$ contains a rational torus, Klein bottle, or Möbius strip, or cylinder with periodic flow. It remains to exclude cylinders with nonperiodic flow. Arguing again by contradiction, suppose that $Z \subset S$ is such a cylinder.

We claim that the support of the measure $\mathfrak{m}$ does not meet $\stackrel{\circ}{Z}=Z \backslash \partial Z$. To see this, pick open neighbourhoods $U_{ \pm} \subset M$ of the boundary orbits $\partial_{ \pm} Z$, and a compact collar neighbourhood $W$ of $Z \backslash\left(U_{+} \cup U_{-}\right)$in $M \backslash\left(U_{+} \cup U_{-}\right)$in which the flow moves from $U_{-}$to $U_{+}$. Pick a flow box $[a, b] \times[-\varepsilon, \varepsilon] \times[-\varepsilon, \varepsilon] \subset W$ with coordinates $(r, x, y)$ around any point $p=(a, 0,0) \in Z \backslash\left(U_{+} \cup U_{-}\right)$on which $h(r, x, y)=h(r)$ and $X=\partial_{x}$. Given a 1-form $\alpha$ and the sequence $r_{n} \searrow a$ from above, we pick $\bar{X}$-orbits $\gamma_{n}$ with $\gamma_{n}(0)=\left(r_{n}, 0,0\right)$ and $\gamma_{n}\left(T_{n}\right)=\left(r_{n}, 0, y_{n}\right)$ for some $y_{n} \in[-\varepsilon, \varepsilon]$. This is possible because the flow on $\left\{r_{n}\right\} \times T^{2}$ is irrational. 
Moreover, we pick the $T_{n} \rightarrow \infty$ large enough so that

$$
\left|\frac{1}{T_{n}} \int_{0}^{T_{n}} \alpha\left(\dot{\gamma}_{n}(t)\right) d t-c_{r_{n}}(\alpha)\right| \rightarrow 0
$$

as $n \rightarrow \infty$. It follows that

$$
\int_{M} \alpha(X) d \mathfrak{m}=\lim _{n \rightarrow \infty} \frac{1}{T_{n}} \int_{0}^{T_{n}} \alpha\left(\dot{\gamma}_{n}(t)\right) d t .
$$

Since the orbits $\gamma_{n}$ always traverse $W$ in some uniformly bounded time, and the time they spend in $U_{ \pm}$tends to infinity as $n \rightarrow \infty$ (because they get closer and closer to the periodic orbits $\partial_{ \pm} Z$ ), the fraction of time the orbit $\gamma_{n}$ spends in $W$ converges to 0 as $n \rightarrow \infty$. Hence the parts of $\gamma_{n}$ in $W$ do not contribute to the limit of the above integral, and thus $\int_{M} \alpha(X) d \mathfrak{m}=0$ if $\alpha$ has support in $W$. Making $U_{ \pm}$arbitrarily small, this proves the claim.

Now we pick any simple closed curve $\delta_{a} \subset \stackrel{\circ}{Z}$ transverse to $X$ and construct a closed 1-form $\alpha$ supported near $\delta_{a}$ as in Step 1. As in Step 1, it follows that $c_{r}(\alpha)=c \neq 0$ for all $r>a$ close to $a$. On the other hand, $\int_{M} \alpha(X) d \mathfrak{m}=0$ because the supports of $\alpha$ and $\mathfrak{m}$ do not intersect. So again we have a contradiction to (10), which concludes the proof of Lemma 3.4.

Proof of Proposition 3.2. Case 1: The direction of $\bar{X}$ is rational. By Lemma 3.4(a), in this case the flow of $\bar{X}$ is also periodic on $S$. Let $\gamma$ be any periodic orbit in $S$ with covering number $d_{\gamma}$ as defined above. The local analysis near $\gamma$ in Lemma 2.4 shows that $d_{\gamma} \gamma$ is homologous to the simple closed orbits on $\{r\} \times T^{2}$ for $r>a$, Hence formula (8), closedness of $\nu$, and the normalization $\int_{\gamma} \nu=1 / d_{\gamma}$ imply for $r>a$ close to $a$ :

$$
c_{-}(\nu)=c_{r}(\nu)=\int_{d_{\gamma} \gamma} \nu=1
$$

The same argument near $r=d$ yields $c_{+}(\nu)=1$.

Case 2: The direction of $\bar{X}$ is irrational. By Lemma 3.4(b), $S_{a}=\bar{V} \cap h^{-1}(a)$ is either an isolated periodic orbit or a torus on which the flow is irrational, and the same holds for $S_{b}=\bar{V} \cap h^{-1}(b)$. If $S_{a}$ is an irrational torus, there is another integrable region $V^{\prime} \subset M \backslash N$ containing $S_{a}$ in its boundary. If the direction of the vector field $X$ on $V^{\prime}$ is constant, then it must be irrational by Lemma 3.4(a). So the other boundary component of $V^{\prime}$ is again a torus with irrational flow or an isolated periodic orbit. Continuing like this in both directions, we find a maximal chain of connected components of $M \backslash N$ on which the direction of $X$ is constant and irrational, meeting along tori with irrational flow. Now we use the remaining freedom in the normalization of $\nu$ on the isolated periodic orbits and irrational tori in Remark 2.9] to arrange $c_{+}(\nu)=c_{-}(\nu)$ throughout this chain as follows: We arbitrarily normalize $\nu$ on the first boundary component and then succesively extend it over the integrable 
regions, thus inducing normalizations on the other boundary components such that the condition $c_{+}(\nu)=c_{-}(\nu)$ holds across this chain. If the first and last boundary components in this chain coincide (this can only happen when both are irrational tori), then the $c_{ \pm}(\nu)$ are the same at all the boundary tori in this chain and thus match also at the ends.

This concludes the proof of Proposition 3.2, and hence of Theorem 1.1

\section{Construction of a smooth counterexample}

In this section we prove Proposition 1.6 from the Introduction by constructing a smooth counterexample.

Consider the upper half plane $\mathbb{H} \subset \mathbb{C}$ with its standard hyperbolic metric (of constant curvature -1$)$ and the isometric action of the Möbius transformations $\operatorname{PSL}(2, \mathbb{R})$. Let $\Gamma \subset \operatorname{PSL}(2, \mathbb{R})$ be a lattice so that $\Sigma=\mathbb{H} / \Gamma$ is a closed hyperbolic surface. Let $\lambda$ be the canonical contact 1-form (restriction of the Liouville form $p d q$ ) on the unit cotangent bundle $S^{*} \Sigma$, whose Reeb vector field $R$ defines the geodesic flow for the hyperbolic metric on $\Sigma$.

Standardizing $\lambda$ near a closed Reeb orbit. We first derive a (partial) standard form for $\lambda$ near a closed Reeb orbit $\gamma$ on $S^{*} \Sigma$.

Lemma 4.1. Each closed Reeb orbit $\gamma \subset S^{*} \Sigma$ possesses a tubular neighbourhood $U \cong D \times S^{1}$ with coordinates $(x, y)$ in the disk $D=\left\{x^{2}+y^{2}<\varepsilon\right\}$ and $z$ in $S^{1}=\mathbb{R} / \mathbb{Z}$ along $\gamma \cong\{0\} \times S^{1}$ in which $\lambda$ has the form

$$
\lambda=\left(T_{0}+y^{2}-x^{2}\right) d z+\lambda_{D}
$$

where $T_{0}=\int_{\gamma} \lambda>0$ and $\lambda_{D}$ is a 1-form on $D$ (depending only on $x$ and $y$ ).

Proof. Consider the closed geodesic $\bar{\gamma}$ on $\Sigma$ corresponding to the Reeb orbit $\gamma$. Let $\tilde{\gamma}$ be a lift of $\bar{\gamma}$ to $\mathbb{H}$. The geodesic $\tilde{\gamma} \subset \mathbb{H}$ defines a 1-parameter subgroup $G$ of the isometry group $\operatorname{PSL}(2, \mathbb{R})$ as follows. We pick a parametrization $\tilde{\gamma}(t)$ of $\tilde{\gamma}$ by arclength and oriented according to $R$. For each $t \in \mathbb{R}$ there exists a unique $\phi_{t} \in \operatorname{PSL}(2, \mathbb{R})$ preserving $\tilde{\gamma}$ and mapping $\tilde{\gamma}(0)$ to $\tilde{\gamma}(t)$. Since $\phi_{s+t}$ and $\phi_{s} \circ \phi_{t}$ both preserve $\tilde{\gamma}$ and map $\gamma(0)$ to $\gamma(s+t)$, they are equal, so $G:=\left\{\phi_{t} \mid t \in \mathbb{R}\right\}$ is a 1-parameter subgroup of $\operatorname{PSL}(2, \mathbb{R})$. The fact that $\tilde{\gamma}$ is the lift of a closed geodesic $\bar{\gamma}$ means that

$$
\Gamma_{G}:=\Gamma \cap G
$$

is a lattice in $G \cong \mathbb{R}$.

Since the projection $\mathbb{H} \rightarrow \Sigma$ is a covering and $\bar{\gamma}$ is closed, each point on $\tilde{\gamma}$ has a neighbourhood that does not intersect any other lift of $\bar{\gamma}$. By a compactness argument, this implies that for $\varepsilon>0$ suffiently small the strip

$$
\widetilde{N}:=\{p \in \mathbb{H} \mid \operatorname{dist}(p, \tilde{\gamma})<\varepsilon\}
$$


around $\tilde{\gamma}$ does not intersect any other lift of $\bar{\gamma}$. After shrinking $\varepsilon$ further, we may thus assume that whenever any two points $p_{1}$ and $p_{2}$ in $\widetilde{N}$ are related by an element $g \in \Gamma$ we have $g \in G$. Let the expressions "to the left/right of $\tilde{\gamma}$ " have the obvious meaning. For $\delta \in(-\varepsilon, \varepsilon)$ we define the curves

$$
\tilde{\gamma}_{\delta}:=\{p \in \mathbb{H}|\operatorname{dist}(p, \tilde{\gamma})=| \delta \mid \text { and } p \text { lies to the left/right of } \tilde{\gamma}\}
$$

where we choose "left" for $\delta<0$ and "right" for $\delta>0$, and $\tilde{\gamma}_{0}:=\tilde{\gamma}$. Since $G$ acts by orientation preserving isometries, the curves $\tilde{\gamma}_{\delta}$ are orbits of $G$. Thus $\widetilde{N} \cong(-\varepsilon, \varepsilon) \times \mathbb{R}$ is a $G$-invariant set on which $G \cong \mathbb{R}$ acts by translation in the $\mathbb{R}$-direction. It follows that the annulus

$$
N:=\tilde{N} / \Gamma=\tilde{N} / \Gamma_{G} \cong(-\varepsilon, \varepsilon) \times S^{1} \subset \Sigma
$$

inherits an isometric action of $G / \Gamma_{G} \cong S^{1}$ by translations in the $S^{1}$-direction. Consider the preimage $\pi^{-1}(N) \cong(-\varepsilon, \varepsilon) \times S^{1} \times S^{1}$ of $N$ in the $S^{1}$-bundle $\pi: S^{*} \Sigma \rightarrow \Sigma$. The isometric $S^{1}$-action on $N$ lifts (by taking differentials) to an $S^{1}$-action on $\pi^{-1}(N)$ preserving the canonical 1-form $\lambda$. Since the action is free, there exists a tubular neighbourhood $U \cong D \times S^{1}$ of $\gamma \cong\{0\} \times S^{1}$ in $\pi^{-1}(N)$ with coordinates $(x, y)$ in the disk $D=\left\{x^{2}+y^{2}<\varepsilon\right\}$ (for a different $\varepsilon>0$ ) and $z$ in $S^{1}=\mathbb{R} / \mathbb{Z}$ in which the action is given by translation in the $S^{1}$-direction. Since the 1 -form $\lambda$ on $U$ is $S^{1}$-invariant, we can write it uniquely as

$$
\lambda=H(x, y) d z+\lambda_{D}
$$

for some function $H$ and some 1 -form $\lambda_{D}$ on $D$. Since $\gamma$ is a Reeb orbit, the Reeb vector field $R$ along $\gamma$ is given by $R(0,0, z)=T_{0}^{-1} \partial_{z}$ for some constant $T_{0}>0$. The conditions $\lambda(R)=1$ and $i_{R} d \lambda=0$ along $\gamma$ now imply that

$$
H(0,0)=T_{0}, \quad d H(0,0)=0 .
$$

In particular, $\int_{\gamma} \lambda=T_{0}$. Now each closed orbit $\gamma$ for the geodesic flow on a hyperbolic surface is hyperbolic (see e.g. [12]). This translates into $H$ having a nondegenerate critical point of index 1 at $(0,0)$ (see the discussion in the next paragraph). So by the Morse Lemma we can choose coordinates $(x, y)$ in which

$$
H(x, y)=T_{0}+y^{2}-x^{2} .
$$

Reeb dynamics for $S^{1}$-invariant contact forms on $D \times S^{1}$. Consider more generally an $S^{1}$-invariant contact form on $U=D \times S^{1}$ given by

$$
\lambda=H(x, y) d z+\lambda_{D}, \quad H(x, y)>0, \quad d H(0,0)=0 .
$$

For sufficiently small $D$, the contact condition is equivalent to $d \lambda_{D}$ being a positive area form on $D$. Since the Reeb vector field $R$ is $S^{1}$-invariant, we can write it uniquely as

$$
R=T(x, y)^{-1} \partial_{z}+R_{D}
$$


for some positive function $T$ and some vector field $R_{D}$ on $D$. We compute

$$
d \lambda=d H \wedge d z+d \lambda_{D}, \quad i_{R} d \lambda=-T^{-1} d H+i_{R_{D}} d \lambda_{D},
$$

so the condition $i_{R} d \lambda=0$ translates into

$$
d H=T i_{R_{D}} d \lambda_{D} .
$$

In other words, $R_{D}$ is the Hamiltonian vector field of the autonomous Hamiltonian function $H$ with respect to the symplectic form $-T d \lambda_{D}$ on $D$. Writing $w=(x, y) \in D$, the equations for the Reeb flow become

$$
\dot{w}=R_{D}(w), \quad \dot{z}=T(w)^{-1} .
$$

Thus the Reeb flow projects onto the Hamiltonian flow of $H$ on $D$, and the $z$-component can be integrated to $z(t)=z(0)+T(w)^{-1} t$. Since $z$ lives in $\mathbb{R} / \mathbb{Z}$, we see that the Reeb orbit starting at time $t=0$ at $(w, 0)$ returns to the slice $D \times\{0\}$ at time $T(w)$ and the Poincaré return map on $D \times\{0\}$ is given by

$$
\phi(w)=\psi_{T(w)}(w),
$$

where $\psi_{t}$ is the flow of $R_{D}$ on $D$ (which may run out of $D$ ) and $T(w)$ is the return time. Note that $d H(0,0)=0$ implies $R_{D}(0,0)=0$, so $(0,0) \in D$ is a fixed point of $\phi$. The linearization of $\phi$ at $(0,0)$ is given by

$$
D \phi(0,0) \cdot w=D \psi_{T_{0}}(0,0) \cdot w+(D T(0,0) \cdot w) R_{D}(0,0)=D \psi_{T_{0}}(0,0) \cdot w,
$$

where $T_{0}:=T(0,0)=H(0,0)>0$. Thus $D \phi(0,0)=D \psi_{T_{0}}(0,0)$ is the time $T_{0}$ map of the linearized Hamiltonian flow

$$
\dot{w}=J S \cdot w, \quad J=\left(\begin{array}{cc}
0 & -1 \\
1 & 0
\end{array}\right)
$$

where $S$ is the Hessian of $H$ at $(0,0)$. A short computation shows that the eigenvalues $\tau$ of $J S$ satisfy $\tau^{2}+\operatorname{det} S=0$. Hence the Reeb orbit $\gamma=\{(0,0)\} \times S^{1}$ is

- degenerate (at least one eigenvalue is zero) iff $\operatorname{det} S=0$, i.e., $(0,0)$ is a degenerate critical point of $H$;

- hyperbolic (both eigenvalues are real and nonzero) iff $\operatorname{det} S<0$, i.e., $(0,0)$ is a nondegenerate critical point of $H$ of index 1 ;

- elliptic (both eigenvalues are imaginary and nonzero) iff $\operatorname{det} S>0$, i.e., $(0,0)$ is a nondegenerate critical point of $H$ of index 0 or 2 .

This fills in the argument used in the proof of Lemma 4.1. For later use, let us record the following consequences of our discussion: 
(i) Closed Reeb orbits in $U$ are of the form $\{w\} \times S^{1}$ for critical points $w$ of $H$.

(ii) Invariant tori for the Reeb flow in $U$ are of the form $H^{-1}(c) \times S^{1}$, where the level sets $H^{-1}(c)$ of $H$ are diffeomorphic to the circle.

In particular, if $\gamma=\{(0,0)\} \times S^{1}$ is hyperbolic and $D$ sufficiently small, then $U$ contains no closed orbits except $\gamma$ and no invariant tori.

Modifying $\lambda$ near a closed Reeb orbit. Now we turn back to the neighbourhood $U$ of a closed Reeb orbit $\gamma$ described in Lemma 4.1, Let $\chi:[0, \varepsilon] \rightarrow$ $[0,1]$ be a monotone cutoff function which equals 0 near 0 and 1 near $\varepsilon$. Set $r^{2}:=x^{2}+y^{2}$. We modify the contact form $\lambda$ on $U$ to

$$
\lambda_{\chi}:=H_{\chi}(x, y) d z+\lambda_{D}, \quad H_{\chi}(x, y):=T_{0}+\chi\left(r^{2}\right)\left(y^{2}-x^{2}\right) .
$$

By the discussion above, $\lambda_{\chi}$ is a contact form. Since it agrees with $\lambda$ near the boundary of $U$, we can extend it by $\lambda$ to a contact form on $S^{*} \Sigma$ that we will still denote by $\lambda_{\chi}$. Note that on the region $\{\chi=0\}$ the form $\lambda_{\chi}$ agrees with $T_{0} d z+\lambda_{D}$, so its Reeb flow is periodic moving in the $z$-direction (in particular, it is integrable) on this region. On the other hand, we have

Lemma 4.2. Every smooth integral of motion for the Reeb flow of $\lambda_{\chi}$ on $S^{*} \Sigma \backslash$ $\{\chi=0\}$ is constant.

Proof. Let us first determine the critical points of $H_{\chi}$ on $D$. At a critical point we have

$$
\begin{aligned}
& 0=\partial_{x} H_{\chi}=2 x \chi^{\prime}\left(r^{2}\right)\left(y^{2}-x^{2}\right)-2 x \chi\left(r^{2}\right), \\
& 0=\partial_{y} H_{\chi}=2 y \chi^{\prime}\left(r^{2}\right)\left(y^{2}-x^{2}\right)+2 y \chi\left(r^{2}\right) .
\end{aligned}
$$

Subtracting $x / 2$ times the first equation from $y / 2$ times the second we obtain

$$
0=\left(y^{2}-x^{2}\right)^{2} \chi^{\prime}\left(r^{2}\right)+r^{2} \chi\left(r^{2}\right) .
$$

Since all terms on the right hand side are nonnegative, this is only possible for $\chi\left(r^{2}\right)=0$. So the only critical level set of $H_{\chi}$ is $\left\{H_{\chi}=T_{0}\right\}$. It consists of the disk $\{\chi=0\}$ of critical points together with the 4 lines $\{x= \pm y\} \backslash\{\chi=0\}$ of regular points. Since $H_{\chi}$ agrees with $H$ near $\partial D$, we obtain a one-to-one correspondence between the level sets of $H$ and those of $H_{\chi}$, the only difference being that the critical point of $H$ at the origin has been replaced by the critical disk $\{\chi=0\}$ for $H_{\chi}$. In view of consequences (i) and (ii) above, this implies a one-to-one correspondence between invariant tori of $H$ and those of $H_{\chi}$ outside the disk $\{\chi=0\}$.

Suppose now that $f: S^{*} \Sigma \backslash\{\chi=0\} \rightarrow \mathbb{R}$ is a nonconstant smooth integral of motion for the Reeb flow of $\lambda_{\chi}$. By the Arnold-Liouville theorem [2], this gives us an open region in $S^{*} \Sigma \backslash\{\chi=0\}$ that is foliated by invariant 2-tori for the 
Reeb flow of $\lambda_{\chi}$. By the preceding discussion, this gives rise to an open region in $S^{*} \Sigma \backslash\{\chi=0\}$ foliated by invariant 2-tori for the Reeb flow of $\lambda$. But this contradicts ergodicity of the geodesic flow on $S^{*} \Sigma$ (see e.g. [12]), hence such an integral of motion cannot exist.

Modifying $\lambda$ near 4 closed Reeb orbits. Now we perform the preceding construction at 4 closed Reeb orbits as follows. Suppose that $\Sigma$ has genus at least 3. Pick four disjoint simple closed geodesics $\bar{\gamma}_{i}$ on $\Sigma$ with corresponding closed Reeb orbits $\gamma_{i}$ and periods $T_{i}=\int_{\gamma_{i}} \lambda, i=1, \ldots, 4$. (Their precise choice will be fixed later.) Let $U_{i} \cong D_{i} \times S^{1}$ be tubular neighbourhoods of $\gamma_{i}$ as in Lemma4.1 (with $T_{0}$ replaced by $T_{i}$ and some $\varepsilon_{i}>0$ ). We modify $\lambda$ as described in (11) on each $U_{i}$, using some cutoff functions $\chi_{i}$, to obtain a new contact form $\tilde{\lambda}$ on $S^{*} \Sigma$. (We could actually take the same $\varepsilon>0$ and the same cutoff function $\chi$ for all $i$, but it will not matter for the following construction.) Recall that in the canonical coordinates $(x, y, z)$ on each region

$$
V_{i}:=\left\{\chi_{i}=0\right\} \subset U_{i}
$$

the form $\tilde{\lambda}$ agrees with $T_{i} d z+\lambda_{D_{i}}$ for some 1-form $\lambda_{D_{i}}$ on the disk $D_{i}=$ $\left\{x^{2}+y^{2}<\varepsilon_{i}\right\}$ such that $d \lambda_{D_{i}}$ is a positive area form. After a modification of $\lambda_{D_{i}}$ on $\left\{\chi_{i}=0\right\} \subset D_{i}$, keeping it fixed near the boundary of $\left\{\chi_{i}=0\right\}$, we may assume that

$$
d \lambda_{D_{i}}=d r \wedge d \phi
$$

in polar coordinates $(r, \phi)$ corresponding to the Cartesian coordinates $(x, y)$ on some annulus

$$
A_{i}:=\left\{r_{i} \leq r \leq r_{i}+\delta\right\} \subset\left\{\chi_{i}=0\right\} \subset D_{i} .
$$

(Note that we use the same $\delta>0$ for all $i=1, \ldots, 4$.) We keep denoting the resulting contact form by $\tilde{\lambda}$. Note that this modification of $\lambda_{D_{i}}$ does not change the Reeb vector field $\tilde{R}$ of $\tilde{\lambda}$, which is still given by $T_{i}^{-1} \partial_{z}$ on $V_{i}$. Recall that a stabilizing 1-form for the vector field $\tilde{R}$ is a 1-form $\nu$ satisfying $i_{\tilde{R}} d \nu=0$ and $\nu(\tilde{R})>0$. The following lemma shows that such forms must be very special.

Lemma 4.3. Let $\nu$ be a stabilizing 1 -form for $\tilde{R}$ on $S^{*} \Sigma \backslash\left(V_{1} \cup \cdots \cup V_{4}\right)$. Then

$$
\nu=c \tilde{\lambda}+\beta
$$

for some constant $c \neq 0$ and some closed 1 -form $\beta$ on $S^{*} \Sigma \backslash\left(V_{1} \cup \cdots \cup V_{4}\right)$.

Proof. Since $i_{\tilde{R}} d \nu=i_{\tilde{R}} d \tilde{\lambda}=0$, we can write $d \nu=f d \tilde{\lambda}$ for a smooth function on $S^{*} \Sigma \backslash\left(V_{1} \cup \cdots \cup V_{4}\right)$ which is invariant under the flow of $\tilde{R}$. By Lemma 4.2 (which clearly continues to hold for $\lambda$ being modified near 4 orbits instead of just one), we must have $f \equiv c$ for some constant $c \in \mathbb{R}$. It follows that $\beta:=\nu-c \tilde{\lambda}$ is closed.

It remains to rule out the case $c=0$. Since $\Sigma$ has genus at least 3 , we find a simple closed geodesic $\bar{\gamma}$ that is disjoint from the simple closed geodesics 
$\bar{\gamma}_{1}, \ldots, \bar{\gamma}_{4}$. Let $\gamma, \gamma^{\prime}$ be the closed Reeb orbits corresponding to $\bar{\gamma}$ and to $\bar{\gamma}$ parametrized backwards, respectively. Rotating at each point of $\bar{\gamma}$ the unit tangent vector to $\bar{\gamma}$ to its opposite yields a cylinder in $S^{*} \sigma$ connecting $\gamma^{\prime}$ to $-\gamma$ (the curve $\gamma$ oppositely oriented). Since the whole cylinder projects onto $\bar{\gamma} \subset \Sigma \backslash\left(\bar{\gamma}_{1} \cup \cdots \cup \bar{\gamma}_{4}\right)$, this shows that

$$
\left[\gamma^{\prime}\right]=-[\gamma] \in H_{1}\left(S^{*} \Sigma \backslash\left(\gamma_{1} \cup \cdots \cup \gamma_{4}\right)\right) \cong H_{1}\left(S^{*} \Sigma \backslash\left(V_{1} \cup \cdots \cup V_{4}\right)\right) .
$$

Now if $c=0$, then $\nu=\beta$ would be closed and thus $\int_{\gamma^{\prime}} \nu=-\int_{\gamma} \nu$, contradicting the condition (which follows from $\nu(\tilde{R})>0$ ) that $\int_{\gamma^{\prime}} \nu$ and $\int_{\gamma} \nu$ must both be positive.

Cut and paste Now we will build a new manifold $M$ out of $S^{*} \Sigma$ by a cut and paste construction. Let us represent a genus 5 surface $\Sigma$ as an iterated connected sum of 5 tori in linear order (in the obvious notation)

$$
\begin{aligned}
\Sigma:=\left(T^{2} \backslash D_{1}^{+}\right) & \cup_{\bar{\gamma}_{1}}\left(T^{2} \backslash\left(D_{1}^{-} \cup D_{2}^{+}\right)\right) \cup_{\bar{\gamma}_{2}}\left(T^{2} \backslash\left(D_{2}^{-} \cup D_{3}^{+}\right)\right) \\
& \cup_{\bar{\gamma}_{3}}\left(T^{2} \backslash\left(D_{3}^{-} \cup D_{4}^{+}\right)\right) \cup_{\bar{\gamma}_{4}}\left(T^{2} \backslash D_{4}^{-}\right) .
\end{aligned}
$$

Note that the gluing curves $\bar{\gamma}_{i}, i=1, \ldots, 4$, on $\Sigma$ are homologous and appear as boundary curves in a pair-of-pants decomposition of $\Sigma$. In view of the FenchelNielsen coordinates on Teichmüller space (see e.g. [14]), there exist hyperbolic metrics on $\Sigma$ making the $\bar{\gamma}_{i}$ geodesics with arbitrarily prescribed positive values of their lengths $T_{1}, \ldots, T_{4}$. We choose a hyperbolic metric such that

$$
T_{2}-T_{1} \neq T_{4}-T_{3}
$$

Note that the lengths $T_{i}$ are equal to the periods $\int_{\gamma_{i}} \lambda$ of the corresponding closed Reeb orbits $\gamma_{i}$ in $S^{*} \Sigma$. We modify $\lambda$ near $\gamma_{1}, \ldots, \gamma_{4}$ to the contact from $\tilde{\lambda}$ as above. Recall that

$$
d \tilde{\lambda}=d \lambda_{D_{i}}=d r \wedge d \phi
$$

in coordinates $(r, \phi, z)$ on the regions

$$
A_{i} \times S^{1} \subset V_{i}, \quad A_{i}:=\left\{r_{i} \leq r \leq r_{i}+\delta\right\} \subset\left\{\chi_{i}=0\right\} \subset D_{i} .
$$

We cut out from $S^{*} \Sigma$ the four solid tori $\left\{r<r_{i}\right\} \subset V_{i}$. Then we glue the collar neighbourhoods of the boundary tori of the resulting manifold pairwise via the orientation preserving diffeomorphism

$\Phi_{12}:\left[r_{1}, r_{1}+\delta\right] \times S^{1} \times S^{1} \rightarrow\left[r_{2}, r_{2}+\delta\right] \times S^{1} \times S^{1}, \quad(r, \phi, z) \mapsto\left(r_{2}+\delta+r_{1}-r,-\phi, z\right)$,

and similarly for $i=3,4$. This yields an oriented, closed manifold $M$. Since the gluing maps preserve the area form $d r \wedge d \phi$, the 2 -form $d \tilde{\lambda}$ descends to a nowhere vanishing closed 2-form $\omega$ on $M$. Now the following lemma concludes the proof of Proposition 1.6. 
Lemma 4.4. There exists a nowhere vanishing vector field $X$ on the above manifold $M$ generating ker $\omega$ with the following properties:

(i) $X$ admits no stabilizing 1-form (note that this is actually a property of $\omega$ );

(ii) $X$ solves the stationary Euler equations (11), (2) for some metric and volume form on $M$.

Proof. We will repeatedly use the following simple observation:

(O) Let $\nu$ be a 1-form stabilizing a vector field $X$ generating the foliation by circles $\{p\} \times S^{1}$ on a connected manifold $P \times S^{1}$ (in our applications $P$ will be a disk or annulus). Then $\int_{\{p\} \times S^{1}} \nu$ does not depend on $p \in P$.

To see this, connect two points $p, q \in P$ by a curve $\gamma$. The condition $i_{X} d \nu=0$ and Stokes' theorem now imply

$$
0=\int_{\gamma \times S^{1}} d \nu=\int_{\{q\} \times S^{1}} \nu-\int_{\{p\} \times S^{1}} \nu .
$$

We first apply this observation to the solid tori $V_{i} \cong\left\{\chi_{i}=0\right\} \times S^{1}$ with the contact form $\tilde{\lambda}=T_{i} d z+\lambda_{D_{i}}$. Let us pick points $w_{i} \in \partial D_{i}$. Then the closed Reeb orbits $\gamma_{i}^{\prime}:=\left\{w_{i}\right\} \times S^{1}$ satisfy

$$
\int_{\gamma_{i}^{\prime}} \tilde{\lambda}=\int_{\gamma_{i}} \tilde{\lambda}=T_{i}
$$

Let now $(M, \omega)$ be as in the lemma, and $X$ be a nowhere vanishing vector field generating ker $\omega$. Consider in $M$ the regions

$$
\begin{aligned}
& M_{12}:=\left(V_{1} \backslash\left\{r<r_{1}\right\}\right) \cup_{\Phi_{12}}\left(V_{2} \backslash\left\{r<r_{2}\right\}\right) \cong[1,2] \times S^{1} \times S^{1}, \\
& M_{34}:=\left(V_{3} \backslash\left\{r<r_{3}\right\}\right) \cup_{\Phi_{34}}\left(V_{4} \backslash\left\{r<r_{4}\right\}\right) \cong[3,4] \times S^{1} \times S^{1} .
\end{aligned}
$$

By construction, $X$ generates the foliation by circles $\{(r, \phi)\} \times S^{1}$ in the $z$ direction on $M_{12}$ and $M_{34}$. Note that

$$
\left(M \backslash\left(M_{12} \cup M_{34}\right), \omega\right) \cong\left(S^{*} \Sigma \backslash\left(V_{1} \cup \cdots \cup V_{4}\right), d \tilde{\lambda}\right) .
$$

In particular, the closed Reeb orbits $\gamma_{i}^{\prime}$ defined above can be viewed as sitting on the boundary components $\{i\} \times S^{1} \times S^{1}, i=1, \ldots, 4$, of $M_{12}$ resp. $M_{34}$.

Now we can prove (i). Recall from the construction of $\Sigma$ that $\bar{\gamma}_{2}-\bar{\gamma}_{1}$ is the boundary of a region $\Sigma_{12} \subset \Sigma$ diffeomorphic to a 2-torus with two disks removed. In particular, $\bar{\gamma}_{1}$ and $\bar{\gamma}_{2}$ are homologous in $\Sigma$. Their lifts to $S^{*} \Sigma$ satisfy

$$
\left[\gamma_{2}\right]=\left[\gamma_{1}\right]-2[F] \in H_{1}\left(S^{*} \Sigma\right)
$$

where $[F]$ is the class of a fibre of the circle bundle $S^{*} \Sigma \rightarrow \Sigma$ and -2 is the Euler characteristic of $\Sigma_{12}$. The analogous argument applied to the region $\Sigma_{34} \subset \Sigma$ bounded by $\bar{\gamma}_{4}-\bar{\gamma}_{3}$ yields

$$
\left[\gamma_{4}\right]=\left[\gamma_{3}\right]-2[F] \in H_{1}\left(S^{*} \Sigma\right) .
$$


(The precise coefficient -2 in front of $[F]$ in (14) and (15) will not matter for us, but it will be important that the coefficient is the same in both equations.) Since the regions $\Sigma_{12}$ and $\Sigma_{34}$ are disjoint, relation (14) continues to hold in $H_{1}\left(S^{*} \Sigma \backslash\left(\gamma_{3} \cup \gamma_{4}\right)\right)$, and relation (15) in $H_{1}\left(S^{*} \Sigma \backslash\left(\gamma_{1} \cup \gamma_{2}\right)\right)$. Replacing the $\gamma_{i}$ by their push-offs $\gamma_{i}^{\prime}$, we obtain the relations

$$
\left[\gamma_{2}^{\prime}\right]-\left[\gamma_{1}^{\prime}\right]+2[F]=\left[\gamma_{3}^{\prime}\right]-\left[\gamma_{4}^{\prime}\right]+2[F]=0 \in H_{1}\left(S^{*} \Sigma \backslash\left(V_{1} \cup \cdots \cup V_{4}\right)\right) .
$$

Suppose now that $\nu$ is a 1 -form on $M$ stabilizing $X$. By Lemma 4.3, on $S^{*} \Sigma \backslash\left(V_{1} \cup \cdots \cup V_{4}\right)$ we have

$$
\nu=c \tilde{\lambda}+\beta
$$

for some constant $c \neq 0$ and some closed 1-form $\beta$ on $S^{*} \Sigma \backslash\left(V_{1} \cup \cdots \cup V_{4}\right)$. Integrating $\nu$ over $\gamma_{1}^{\prime}, \ldots, \gamma_{4}^{\prime}$ and using (16) and (13), we obtain

$$
\begin{gathered}
\int_{\gamma_{2}^{\prime}} \nu-\int_{\gamma_{1}^{\prime}} \nu=c\left(T_{2}-T_{1}\right)-2 \int_{F} \beta, \\
\int_{\gamma_{4}^{\prime}} \nu-\int_{\gamma_{3}^{\prime}} \nu=c\left(T_{4}-T_{3}\right)-2 \int_{F} \beta .
\end{gathered}
$$

Due to condition (12) on the $T_{i}$ (and since $c \neq 0$ ), the right hand sides of (17) and (18) are not equal. On the other hand, applying the observation (O) above to the regions $M_{12}$ and $M_{34}$, we conclude that the left hand sides of (17) and (18) are both zero. This contradiction proves (i).

For (ii), we need to construct a nowhere vanishing vector field $X$ on the manifold $M$ generating ker $\omega$ such that

$$
i_{X} d \lambda=-d h, \quad \lambda(X)>0
$$

for some 1-form $\lambda$ and function $h$ on $M$. (One should not confuse $\lambda$ with the canonical contact form on $S^{*} \Sigma$, which will not be used any more. It was explained in the Introduction how to recover from these data a volume form and metric for which $X$ satisfies the stationary Euler equations (11), (2).)

On $M \backslash\left(M_{12} \cup M_{34}\right)$ we take $\lambda:=\tilde{\lambda}, X:=\tilde{R}$ its Reeb vector field, and $h:=0$, so $i_{X} d \lambda=0$ and $\lambda(X)=1$ on this region. Note that we can extend $(\lambda, X, h)$ slightly into $M_{12}$ and $M_{34}$ by the same formulas (we can actually extend them up to the region where the gluing happens). Recall that the kernel foliation of $\omega$ on the region $M_{12} \cong[1,2] \times S^{1} \times S^{1}$ consists of the circles $\{(r, \phi)\} \times S^{1}$ in the $z$-direction. By construction of $\tilde{\lambda}$, the given data are equal to

$$
\lambda=T_{i} d z+\lambda_{D_{i}}, \quad X=T_{i}^{-1} \partial_{z}, \quad h=0
$$

near the boundary component $\{i\} \times T^{2}, i=1,2$. We extend $\lambda$ and $X$ over $M_{12}$ satisfying (19) as follows (the extension over $M_{34}$ is analogous).

Let $b$ be a positive function on $[1,2]$ which is constant $T_{1}$ near 1 , constant $T_{2}$ near 2 , and has regions with positive derivative as well as regions with negative 
derivative. Let $g$ be a positive function on $[1,2]$ which is constant $T_{1}^{-1}$ near 1 , constant $T_{2}^{-1}$ near 2 , and such that

$$
\int_{1}^{2} g(r) b^{\prime}(r) d r=0
$$

(This is possible because $b^{\prime}$ changes signs.) We pick any 1 -form $\lambda_{D}$ on $[1,2] \times S^{1}$ (depending only on $r$ and $\phi$ ) which agrees with $\lambda_{D_{i}}$ near $\{i\} \times S^{1}, i=1,2$. Now we define the extension over $[1,2] \times T^{2}$ by

$$
\lambda:=b(r) d z+\lambda_{D}, \quad X:=g(r) \partial_{z}, \quad h(r):=\int_{1}^{r} g(s) b^{\prime}(s) d s .
$$

The choice of $b$ and $g$ ensures that this matches the given data near the boundary (condition (20) ensures that $h(r)=0$ near $r=2$ ). Note that $X$ generates ker $\omega$. Since

$$
i_{X} d \lambda=i_{g(r) \partial_{z}} b^{\prime}(r) d r \wedge d z+i_{g(r) \partial_{z}} b^{\prime}(r) d \lambda_{D}=-g(r) b^{\prime}(r) d r+0=-d h
$$

and $\lambda(X)=b(r) g(r)>0$, the triple $(\lambda, X, h)$ satisfies (19). This concludes the proof of Lemma 4.4, and hence of Proposition 1.6.

\section{References}

[1] J. An and Z. Wang, Curve Selection Lemma for semianalytic sets and conjugacy classes of finite order in Lie groups, arXiv:math/0506160.

[2] V.I. Arnold, Sur la géométrie differentielle des groupes de Lie de dimension infinie et ses applications à lhydrodynamique des fluides parfaits, Ann. Inst. Fourier 16, 316-361 (1966).

[3] V.I. Arnold and B. Khesin, Topological Methods in Hydrodynamics, Springer (1998).

[4] F. Bourgeois, Y. Eliashberg, H. Hofer, K. Wysocki and E. Zehnder, Compactness results in symplectic field theory, Geom. Topol. 7, 799888 (2003).

[5] F. Bruhat and H. Whitney, Quelques propriétés fondamentales des ensembles analytiques-réels, Comment. Math. Helv. 33, 132-160 (1959).

[6] K. Cieliebak and E. Volkov, First steps in stable Hamiltonian topology, Journal of the European Mathematical Society, to appear.

[7] J. Etnyre and R. Ghrist, Contact topology and hydrodynamics I: Beltrami fields and the Seifert conjecture, Nonlinearity 13, no. 2, 441-458 (2000). 
[8] J. Etnyre and R. Ghrist, Contact topology and hydrodynamics III: Knotted flowlines, Trans. Amer. Math. Soc. 352, 5781-5794 (2000).

[9] J. Etnyre and R. Ghrist, Generic hydrodynamic instability for curl eigenfields, SIAM J. Appl. Dynamical Systems 4 (2), 377-390 (2005).

[10] S. Friedlander and M. Vishik, Instability criteria for steady flows of a perfect fluid, Chaos 2, no. 3, 455-460 (1992).

[11] M. Goresky and R. MacPherson, Stratied Morse Theory, Springer (1988).

[12] B. Hasselblatt and A. Katok, Introduction to the modern theory of dynamical systems, Cambridge University Press (1995).

[13] H. Hofer, K. Wysocki and E. Zehnder, The Dynamics on a strictly convex energy surface in $\mathbb{R}^{4}$, Annals of Mathematics 148, 197-289 (1998).

[14] C. Hummel, Gromov's compactness theorem for pseudo-holomorphic curves, Birkhäuser (1997).

[15] M. Hutchings and C. Taubes, The Weinstein conjecture for stable Hamiltonian structures, Geom. Topol. 13, no. 2, 901-941 (2009).

[16] D. Sullivan, Cycles for the dynamical study of foliated manifolds and complex manifolds, Invent. Math. 36, 225-255 (1976).

[17] D. Sullivan, A foliation of geodesics is characterized by having no tangent homologies, J. Pure and Appl. Algebra 13 (1978), 101-104. 